\title{
Spiel um Zeit
}

\section{Temporale Konstellationen des Vergehens in zeitgenössischer Fotografie, Literatur und theatraler Installation}

\begin{abstract}
Im Zentrum des Vanitas-Diskurses stehen temporale Relationen von Anund Abwesenheit, die in der zeitgenössischen Kunst als Spiel zwischen Permanenz und Metamorphose verhandelt werden. Ausgehend von der künstlerischen Zeitreflexion des Medienkünstlers Helmut Wimmer und Hélène Cixous' literarischer Konstruktion einer achronisch verfassten All-Zeit beleuchtet der Beitrag anhand zweier theatral-installativer Arbeiten von Forced Entertainment und Romeo Castellucci diese signifikante zeitgenössische Strategie zur Thematisierung von Vergänglichkeit: Errichtet werden temporale Gefüge, die das Vergehen von Zeit als spezifische Konstellation von Dauer thematisieren und so die Dialektik von Ewigkeit und Vergängnis performativ ausloten.
\end{abstract}

\section{Der Abgrund des Denkens}

Beginnt man einmal das Denken über Sterben und Tod, über Werden und Vergehen des Lebens, über die Spannung von Endlichkeit und Ewigkeit, so werden Auge und Ohr, solcherart angeregt, geradezu überall auf bildliche Symbole und sprachliche Chiffren der Vergänglichkeit gelenkt. Dann weist die Rede vom Windhauch, von verwelkenden Blumen oder von Rauch ebenso über das Reich der profanen Naturerscheinungen hinaus wie die Bilder von Seifenblasen, Uhren oder Pfeifen zu Indizes eines symbolisch verschlüsselten Todesbedenkens werden. Als bildspendende Referenz derartiger Codierungen entpuppt sich umgehend die vielfältige frühneuzeitliche Vanitas-Topik. Die Rezeption des alttestamentlichen Buches Kohelet hat dort die Reflexion über die Vergänglichkeit variantenreich als Klage über Verlust und Sinnlosigkeit des Lebens, als Mahnung zur Sinnstiftung jenseits des Besitzerwerbs oder Appell zur Freude am Augenblick hervorgebracht. Der Vanitas-Diskurs hat zugleich das Bewusstsein über die entschwindende Lebenszeit nachhaltig in das semantische Feld des ,Entzugs‘ eingetragen. Die durch die deutsche Einheitsübersetzung bekannte Übertragung des im Kohelet erwähnten hebräischen häväl in das fasslich-unfassbare Sprachbild des Windhauchs (,Windhauch, Windhauch, sagte Kohelet, Windhauch, Windhauch, das ist alles Windhauch“; Koh 1,2 ${ }^{\mathrm{EU}}$ ) koppelt die mit dem Bedeutungsrepertoire der Vanitas verbundenen vielfältigen Vorstellungen von Eitelkeit, Nichtigkeit, Vergänglich-

Ә Open Access. () 2021 Johanna Zorn, publiziert von De Gruyter. (c) BY-NC-ND Dieses Werk ist lizensiert unter einer Creative Commons Namensnennung-Nicht-kommerziell-Keine Bearbeitung 4.0 International Lizenz. https://doi.org/10.1515/9783110716016-013 
keit, Sinnlosigkeit und Scheinhaftigkeit expressis verbis an die Idee des Ephemeren, Unstofflichen, Substanzlosen. ${ }^{1}$

Kein Ding also und doch eine der größten Sachen - die Metapher des Windhauchs sagt schlicht, dass das Sein nur mit der und durch die Zeit zu begreifen, dort zugleich aber gerade nicht als Objekt greifbar, nicht materialiter zu fassen ist. Diese weit über das frühneuzeitliche Bewusstsein hinausreichende Gewissheit, wonach allein die Zeitlichkeit „der Seinsfrage ihren Horizont geben“2 kann, gehört zwar offensichtlich dem Bereich eines immer schon intuitiv oder implizit vorhandenen Wissens an, das in der Alltagsrede über die conditio humana kaum Anlass zur weiteren Beschäftigung bietet, doch hebt sie das Denken aufgrund ihrer vordergründigen Tautologie auch allzu leicht aus den Angeln. Die dominanten zeitlichen Konfigurationen in den künstlerischen Thematisierungen der Vanitas artikulieren eine ebenso naheliegende wie komplizierte Verflechtung von Sein und Zeit, die sich nicht entwirren lässt. Sein erscheint dabei stets als das transitorische ,Jetzt-gerade-nicht-mehr' und in diesem temporalen Modus entfacht es in barocken Darstellungen einen zweifachen Sinn. Einerseits wird Gegenwart explizit auf die Zukunft hin projiziert, die sich als Antizipation des Todes immer schon in den Moment einschreibt, und so als „key element of the vanitas topos“33 fungiert. Andererseits invertiert Gegenwart im Versuch, sie festzuhalten, je in Vergangenheit.

Ein Zeitkonzept, das Bewusstsein über das entschwindende nunc currens stiftet, ${ }^{4}$ steht folglich paradigmatisch für Vanitas thematisierende Kunst. In diesem Sinne gerät etwa das in barocken Stillleben programmatische Stillstellen

1 Vgl. hierzu die umfassenden Ausführungen von Dorothea Scholl. „,Vanitas, vanitatum et omnia vanitas'. Das Buch Kohelet in der europäischen Renaissance- und Barocklyrik und Emblematik“. Bibeldichtung. Hrsg. von Volker Kapp und ders. Berlin: Duncker \& Humboldt, 2006. 221-260, insbesondere S. 223-225.

2 Jacques Derrida. „Ousia und Gramme. Notiz über eine Fußnote“. Sein und Zeit. Randgänge der Philosophie. Übers. von Gerhard Ahrens. Hrsg. von Peter Engelmann. Wien: Passagen, 1999. 57-92, S. 57.

3 Claudia Benthien. „,Vanitas, vanitatum et omnia vanitas‘. The Baroque Transience Topos and its Structural Relations to Trauma“. Enduring Loss in Early Modern Germany. Cross Disciplinary Perspectives. Hrsg. von Lynne Tatlock. Leiden und Boston: Brill, 2010. 51-69, S. 56.

4 Vgl. hierzu die einschlägige Unterscheidung zwischen dem irdischen als gleichsam laufendem Jetzt und dem göttlichen als fortdauerndem Jetzt bei Boethius: „[N]ostrum ,nunc‘ quasi currens tempus facit et sempiternitatem, divinum vero ,nunc ' permanens neque movens sese atque consistens aeternitatem facit;“ Anicius Manlius Severinus Boethius. „De Trinitate“. Theological Tractates. The Consolation of Philosophy. Übers. und hrsg. von Hugh Fraser Stewart u. a. Cambridge, MA: Harvard University Press, 1973. IV, 72-74. 
von Zeit zu einem Vexierspiel, das den „verborgenen betrug“5 ${ }^{* 5}$ momenthaften Eindrucks offen ausstellt. Charakteristisch wird dabei die gegenläufige Kontrastfigur von Halten und Entgleiten, indem die Schönheit von Blumen in ihrer vollen Blüte den bevorstehenden Verfall performiert. Die barocke Lyrik wiederum arbeitet das Kippmoment einer Emphatisierung des Augenblicks (carpe diem), ${ }^{6}$ die vom Eingedenken der Sterblichkeit (memento mori) unmittelbar abhängt, vornehmlich mit antithetischen Sprachbildern durch. Die Musik schließlich wird wegen ihrer Eigenschaft, im Moment des Erklingens sofort wieder zu verklingen, gar zum exemplarischen Sujet barocker Vanitas-Allegorien. ${ }^{7}$ Die unmittelbar einleuchtende, verstandesmäßig allerdings nur schwer zu durchdringende Frage nach dem darin verschleierten spezifischen Verhältnis von An- und Abwesenheit, von Gegenwärtigsein und Verschwinden löst nicht nur Trauer und Melancholie aus. Sie öffnet einen erkenntnistheoretischen und wahrnehmungsästhetischen Abgrund, in den man unversehens blickt, sobald man die sich wechselseitig bestimmenden Größen ,Sein' und ,Zeit‘ nach ihrer Bedeutung und Beziehung befragt.

Die bestimmte Unmöglichkeit, den beiden Vorstellungskomplexen von ,Sein“ und ,Zeit‘ eine dinghafte Existenz zuzuschreiben, hat der Zeitphilosoph Martin Heidegger gar zu ,der großen Sache des Denkens‘ dynamisiert. „Nirgends unter den Dingen finden wir das Sein“" ${ }^{\text {“ }}$ schreibt er. Denn jedes Ding hat zwar „seine Zeit. Sein aber ist kein Ding, ist nicht in der Zeit. "9 Umgekehrt meint das Zeitliche „das Vergängliche, solches, was im Verlauf der Zeit vergeht. [...] Aus der Ständigkeit des Vergehens der Zeit spricht Sein. Gleichwohl finden wir die Zeit nirgends

5 Jacob Grimm und Wilhelm Grimm. [Art.] „Vexierbild“. Deutsches Wörterbuch von Jacob Grimm und Wilhelm Grimm. http://www.woerterbuchnetz.de/DWB?lemma=vexierbild (Zugriff am 09.11.2020).

6 Claudia Benthien und Victoria von Flemming fassen den für das frühneuzeitliche VanitasDenken wesentlichen carpe-diem-Appell explizit als „Emphatisierung der Gegenwart“. Claudia Benthien und Victoria von Flemming. „Einleitung“. Paragrana. Internationale Zeitschrift für Historische Anthropologie 27.2 [Themenheft ,Vanitas. Reflexionen über Vergänglichkeit in Literatur, bildender Kunst und theoretischen Diskursen der Gegenwart‘. Hrsg. von dens.] (2018): 11-35, S. 16.

7 Die prozesshafte Verfassung des Klangs beförderte nicht lediglich die historisch einflussreiche Parallelisierung von Musik und Vergänglichkeit seit der Frühen Neuzeit, sondern brachte ihr zugleich jene kunstphilosophische Überhöhung als wahrer Philosophie ein, die in der Romantik ihren Höhepunkt erreichte. Bereits bei Adam von Fulda heißt es: „Nam musica est etiam philosophia, sed vera philosophia, meditatio mortis continua“. Adam von Fulda. „Musica“ [1490]. Scriptores ecclesiastici de musica sacra potissimum 3. Hrsg. von Martin Gerbert. Sankt Blasien: Typis San-Blasianis, 1784. 329-381, S. 335.

8 Martin Heidegger. „Zeit und Sein“ [1962]. Zur Sache des Denkens. Gesamtausgabe 14. Hrsg. von Friedrich-Wilhelm von Herrmann. Frankfurt a. M.: Vittorio Klostermann, 2007. 3-30, S. 7. 9 Ebd. 
vor als etwas Seiendes wie ein Ding. “10 Dieser fundamentale ontologische Entzug, der das Sein und die Zeit gleichermaßen betrifft, bindet die beiden existentiellen Schlüsselbegriffe so sehr aneinander, dass sie nur als Verhältnis, als „Sachverhalt ${ }^{\text {*11 }} \mathrm{zu}$ denken sind. Dieser droht, sich immer dann aufzulösen, wenn man den einen Begriff vom anderen her zu durchdringen versucht. Jeden propositionalen Sprechakt zur Bestimmung von Sein und Zeit lässt er dabei unweigerlich misslingen und torpediert überdies die Bildfähigkeit des Seins in der Zeit.

Einerseits zeigt sich das Sein als in, mit und durch Zeit sich notwendig Entziehendes, doch zugleich ruft die temporale Struktur des Seins wie selbstverständlich die Idee von Anwesenheit auf, die mit den zum Vanitas-Komplex des Windhauchs konträren, ja schier unvereinbaren Qualitäten von „Dauer und Beständigkeit, Nähe und Verfügbarkeit“ verbunden ist, da sie die „Gegenwart in Form der Vorhandenheit“ denkt. ${ }^{12}$ In dieser Weise hat schon die antike Ontologie den Sinn von Sein wesentlich als ousia oder parousia verstanden und Seiendes „in seinem Sein als ,Anwesenheit‘ gefaßt““13. Selbst die abendländische Präsenzmetaphysik, die Evidenz und Wahrheit unmittelbar an die Gegenwart, an das Jetzt bindet, konnte unterdessen allerdings nicht vergessen, dass nicht nur das Sein, sondern auch die Zeit selbst vergeht und durch diese Vergänglichkeit erst als Zeit besteht. So erscheinen Sein und Zeit beide in ihren je widersprüchlichen und doch komplementären Bedeutungshorizonten wohl als die radikalsten Formen von „Auto-Antonymie“"14: Sie sind Januswörter, die sich zu sich selbst semantisch gegensätzlich verhalten und auf diese Weise im Spiel der Selbstdifferenz Widerstand gegen ihre Bedeutung leisten. Vergleichbar dem Begriff ,Quantensprung‘, der sowohl die kleinstmögliche Zustandsänderung als auch die größtmögliche Veränderung bezeichnet, erscheint das Sein immer kontradiktorisch zugleich als ,Jetzt‘ und ,Nicht-Mehr'. Solche Aporetik von Anwesenheit und Entzug hält das ontologische Denken des Seins in der Zeit gefangen, macht aus dem ambivalenten Kompositum ,Sein-als-Zeit‘ zugleich aber offensichtlich auch eine der produktivsten und weitreichendsten Rätselgestalten der Kunst.

10 Ebd.

11 Ebd., S. 8.

12 Derrida 1999 (Anm. 2), S. 58.

13 Martin Heidegger. Sein und Zeit. 19. Aufl. Tübingen: Niemeyer, 2006 [1927], S. 25.

14 Andreas Blank. Prinzipien des lexikalischen Bedeutungswandels am Beispiel der romanischen Sprachen. Tübingen: Niemeyer, 1997, S. 225. 


\section{Zeit-Schichtungen: Fotografische Memoriale des künftig Vergangenen}

Von der eigentümlichen Schwierigkeit, die temporale Spannung von An- und Abwesenheit im (Sprach-)Bild einzufangen, künden daher auch eine Vielzahl von zeitgenössischen Rekursen auf den Darstellungskomplex der Vanitas. ${ }^{15} \mathrm{Im}$ Jahr 2018 etwa hat der Medienkünstler Helmut Wimmer den Einbruch der Naturgewalten in die Räume von Kunst und Kultur effektvoll inszeniert. Unter dem apokalyptischen Titel The Last Day arrangierte er zwölf fotografische Tableaus, auf denen die Natur in die Säle des Kunsthistorischen Museums Wien eindringt. Auf den Fotografien ereignen sich regelrechte Naturkatastrophen in domestizierten Kulturräumen: Bäume wachsen aus dem Boden, Tiere bevölkern die Räume, Wasserfälle brechen herein, Eis breitet sich aus, Felsen schlagen ein - doch die Anwesenden scheinen davon unberührt, sie betrachten die Bilder unbewegt, während sich die Umgebung verändert, ihr eigenes Enden droht. „[D]er einsame Besucher bleibt, das Eindringen der Natur nicht gewahrend, in die Kontemplation eines Kunstwerks versunken." ${ }^{16}$ (Abb. 1) Am aktuellen historischen Höhepunkt des Anthropozäns, an dem der Mensch als zerstörerischer Einflussfaktor biologische, geologische und meteorologische Prozesse in globalem Ausmaß aus dem Gleichgewicht bringt, kommt der Reflexion über das Ende der Welt, wie wir sie kannten oder zu kennen glaubten, sowie der immer schon zu schnell verrinnenden humanen und terrestrischen Lebenszeit im Kontext der Kunst eine bedeutende Rolle zu. Der Fortschrittsgedanke als Bild des permanenten Wandels kippt dabei zunehmend in Untergangsszenarien um, die mit einer ebenso ironischen wie bedenkenswerten Pointe aufwarten. Diese liegt darin, dass die Unterwerfung der Natur durch das Subjekt als Überwältigung des Menschen durch Naturgewalten radikal zurückschlägt. Das prozesshafte Entwicklungsgeschehen der langsam wachsenden Natur überführen die Fotografien in eine Plötzlichkeit ihres Erscheinens, die unbemerkt bleibt.

Sowohl Sujet als auch künstlerisches Verfahren der Werkserie Wimmers rufen unverkennbar die intermediale Figur der barocken Vanitas auf. Im Zentrum der Arbeit steht eine kommende fundamentale Transformation bekannter Räume, die wieder neue (posthumane) Lebensformen erfordert und ermöglicht.

15 Vgl. hierzu exemplarisch Claudia Benthien und Victoria von Flemming (Hrsg.). Themenheft ,Vanitas. Reflexionen über Vergänglichkeit in Literatur, bildender Kunst und theoretischen Diskursen der Gegenwart' Paragrana. Internationale Zeitschrift für Historische Anthropologie 27.2 (2018). 16 Wolfgang Welsch. „Nach dem Ende des Anthropozäns. Künstlerische Vermutungen“. Kunstforum International 265 (2020): 174-191, S. 186. 


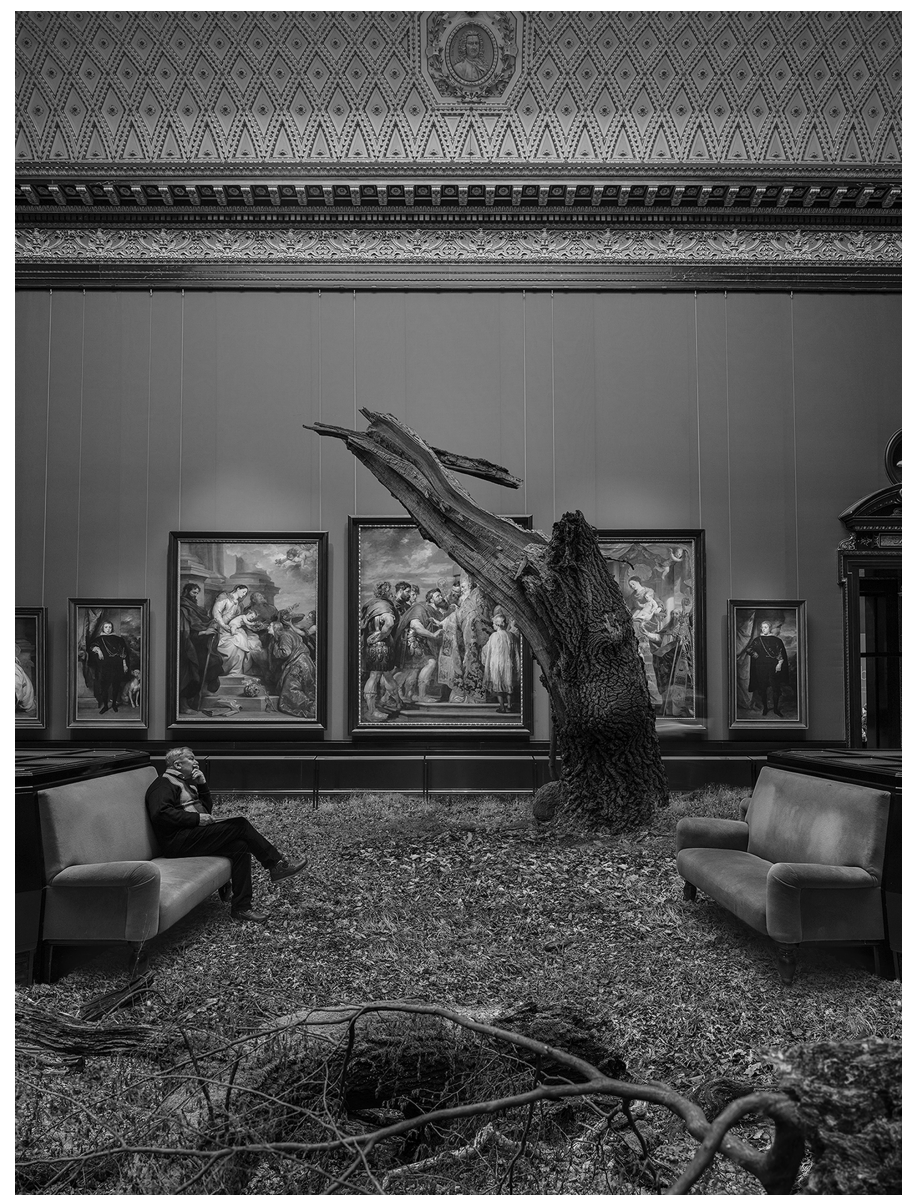

Abb. 1: Helmut Wimmer: The Last Day, Bild Nr. 8, „Mann mit Baumstamm“, C-Print auf Aluminium, 2018, $135 \times 100 \mathrm{~cm}$.

Die bestürzende Wirkung des Werkzyklus ergibt sich aus der Überlagerung eines Zeit-Raums mit anderen, neuen Potentialitäten desselben, und das heißt zugleich, mit dem, was war und dem, was werden könnte. Darstellungsästhetisch signifikant ist das künstlerische Verfahren von temporaler Schichtung. Es handelt sich um das Überformen eines Moments durch einen anderen Zustand, der den Ausgangspunkt keineswegs verschwinden, sondern, im Gegenteil, durchscheinen lässt. Permanenz 
und Metamorphose jenseits des Fortschritts, energeia und dynamis, ${ }^{17}$ verschränken sich durch die digitale Technik der Simulation. Damit ist ein hybrides Zeitkonzept im virtuellen Bild inszeniert, das Veränderung und Verfall des Gegenwärtigen nicht als mehrdeutige Leerstelle bestehen lässt, sondern als Konflikt der Zeiten, aber auch der Mächte und Kräfte von Natur und Kultur ,in ein` Bild setzt.

Exemplarisch präsentiert die Fotoserie The Last Day also eine mediale Strategie, die das Verwandlungsgeschehen des Lebens als antithetische Interferenz von Bildkomplexen inszeniert. Die historisch bedeutsame Bildgattung des Vanitas-Stilllebens erweitert sie dabei um die prozessual-geschichtete Mediatisierung einer Gleichzeitigkeit des Gegensätzlichen und schreibt die Potenz der Zukunft (dynamis) in die Gegenwart ein. Während etwa in Vanitas-Stillleben die Darstellung von Blumen in ihrer vollen Blüte meist die temporale Funktion erfüllt, den kommenden Verfall anzudeuten, die Embleme der Vergänglichkeit also darauf verweisen, dass Zeit in ihnen keineswegs ,stillsteht', inszeniert Wimmer durchgestaltete Lebensräume, die stärker von Zukünftigem bereits tangiert sind und dadurch umgekehrt ,Geschichte haben“. Als solcherart „drastische Bilder voller Kontraste“"18 verweisen die Tableaus im intermedialen Raum zweifelsohne auch auf ein entscheidendes poetologisches Spezifikum barocker Vanitas-Lyrik. Mit Dorothea Scholl ergeht dort nämlich aus dem Hinweis auf die notwendige Verwandlung des Bestehenden der Aufruf zum Nachdenken über die Tiefen des Seins. Das Sonett „Es ist alles eitel“ (1637) von Andreas Gryphius etwa lässt sich mit seiner streng gegenläufigen Struktur als literarische Folie dieses zeitgenössischen Menetekels betrachten. Dieses poetische Mittel der Kontradiktion bewirkt erst, wie Claudia Benthien hervorhebt, den zentralen „thematic content of the poem“, nämlich die „experience of suddenness, of fleeting time“:: ${ }^{19}$ „Was dieser heute bawt | reist jener morgen ein“, „[w]as izt so prächtig blüht | wird bald zutretten werden:“, „[i]tzt scheint des Glückes Sonn | bald donnerts mit beschwerden.“20

Die charakteristische Kompositionstechnik, d. h. das mediale Vermögen der Schichtung von Zeiten, transformiert die historische Signatur unterdessen. Die ba-

17 Vgl. hierzu Aristoteles. Metaphysik. Übers. und hrsg. von Franz F. Schwarz. Stuttgart: Reclam, 1970, S. 307-312, bes. Abschnitt XII 5-6, 1071a-1072a. Aristoteles trifft eine Unterscheidung zwischen energeia, d. h. dem Akt oder der Wirklichkeit, und dynamis, der Potenz oder dem Vermögen. 18 Scholl 2006 (Anm. 1), S. 238.

19 Benthien 2010 (Anm. 3), S. 56.

20 Andreas Gryphius. „VANITAS, VANITATUM, ET OMNIA VANITAS“. Gesamtausgabe der deutschsprachigen Werke 1. Hrsg. von Marian Szyrocki und Hugh Powell. Tübingen: Niemeyer, 1963. 7-8. 
rocke „Zeichenschrift der Vergängnis“21 wird unter den Vorzeichen der digitalen Medientechnik in die offenkundige Kollision von Kultur und Natur, von Topie und Dystopie verwandelt. Zugleich weicht die signifikante lyrische „Ruinen-Symbolik“22 barocker Vanitas einer zeitgenössischen Darstellungspraxis der Schichtung. Wo es etwa in Christian Hoffmann von Hoffmannswaldaus „Die allgemeine Vergänglichkeit“ (ca. 1670) heißt: „Was angeanckert schien dem Mittel-Punkt der Erden, | Verfiel zu seiner Zeit und must ein Unding werden“ ${ }^{23}$, ist das globale Untergangsszenario hier in eine zyklische Struktur des potentiell Wiedererstehenden eingebunden. Denn aus den Ruinen der kulturellen Stätten drängen mit der Aneignung der Räume durch Tiere und Gewächse zugleich neue Formen der Kulturalisierung. Solcherart erfährt der von Walter Benjamin so bezeichnete „barocke Kultus der Ruine“ 24 , der das Leben als Prozess des fortlaufenden Verfalls ausstellt, eine entscheidende Perspektivverschiebung, die den Verlust der Heilsgewissheit ins Bild setzt: Keine Verachtung des Irdischen (contemptus mundi) als Antwort auf das ein für alle Mal eintretende Ende wird hier inszeniert. Nicht der unwiederbringlichen Entrückung des Lebenden aus dem Zentrum der Welt hinaus, nicht den ,Undingen“ der Vergangenheit, sondern all jenem, was gerade jetzt zerstört wird und in Zukunft verschwinden kann, gilt die Aufmerksamkeit. Auf diese Weise gerät die Bilderserie in ihrem Aussagegehalt eigentümlich ambivalent. Einerseits ist sie ostentativer Appell zur Intervention in den globalen ökologischen Zusammenbruch. Andererseits geschieht die menschliche (Selbst-)Vernichtung vor dem Hintergrund eines kosmologischen Zeitkonzepts, in dem das Vergehen und Werden von Lebensformen aus der Schichtung von Zeiten und Räumen erwächst.

Sichtbar wird daran, wie das im Barock sinnfällige, antithetisch strukturierte ,Zeit-Bild‘ zur simultanen Formation von gegenläufigen temporalen Zuständen gerät. Die Interferenz von Zeiten erfolgt nunmehr nach der Logik eines medialen Eingriffs. Der notwendige Lauf der Dinge wird nicht antizipativ aufgerufen und als Abwesendes, zukünftig Eintretendes verschlüsselt, sondern Verfall tritt als Vorwegnahme, als Störung des Jetzt im Bild auf. Dieses spezifische ,Regime des Sichtbaren' lässt das solcherart zukünftig Vergangene in der mediatisierten Unter-

21 Walter Benjamin. „Ursprung des deutschen Trauerspiels“. Gesammelte Schriften I,1. Hrsg. von Rolf Tiedemann und Helmut Schweppenhäuser. Frankfurt a. M.: Suhrkamp, 1980. 203409, S. 353.

22 Scholl 2006 (Anm. 1), S. 230.

23 Christian Hof[f]mann von Hof[f]mannswaldau. „Die allgemeine Vergänglichkeit“. Gedichte. Hrsg. von Manfred Windfuhr. Stuttgart: Reclam, 1964. 103-107, S. 105.

24 Benjamin 1980 (Anm. 21), S. 354. 
gangsvision nicht einfach verschwinden, ${ }^{25}$ sondern in augenfälliger Weise als Mahnung, als memento mori durch die überlagerten Schichten hindurch scheinen. Damit opponiert es zuletzt auch gegen das Verdikt Roland Barthes', wonach die Fotografie „ohne Zukunft“ ${ }^{26}$ sei. Gegenwart invertiert nämlich in die Gedenkstätte des künftig Vergangenen. Die einstmals „vollendete Wirklichkeit“27 (entelecheia) steht hier als medientechnisch inszeniertes Memorial von Vergänglichkeit im Raum. Schwenkt man den Blick von diesem globalen fotografischen Schichtungskonzept hin zu einem Beispiel eines intimen, literarisch inszenierten ZeitRaums, so wird deutlich, welch unterschiedlichen Strategien die zeitgenössischen künstlerischen Bespiegelungen des Vergänglichen folgen.

\section{Literarische Duration: Wiederholung und All-Raum}

In ihrem Buch Meine Homère ist tot... (2014) schreibt sich die Autorin Hélène Cixous im Modus der Autofiktion in den Prozess des Sterbens ihrer Mutter ein, mehr noch, sie stellt die Stimmen von Mutter und Tochter einander gegenüber, schichtet die eigene Stimme über diejenige ihrer Mutter, vertauscht die Rollen und sogar die Zeiten, indem sie die Mutter als eine anruft, die erst in der Zukunft gewesen sein wird. Im Prolog heißt es entsprechend:

Dieses Buch ist bis zur letzten Zeile von meiner Mutter schon geschrieben. Während ich es kopiere siehe da schreibt es sich anders, kommt von der mütterlichen Nacktheit ab ohne dass ich das will, büßt Heiligkeit ein, und wir können nichts dafür noch etwas dazu tun.

Ich beschließe in diese Maman nicht gehorchende Konstruktion ihrer heiligen Schlichtheit entnommene Seiten einzulegen. Das Buch par excellence wäre voll mit Büchern und jenen magischen Fotos die man unter dem Blick eines leidenschaftlichen Lesers zum Leben erwachen sieht, es würde sich öffnen auf Städte hin die zu anderen Städten führen wo meine Mutter geweilt haben wird. Meist sieht man meine Mutter auf der einen Seite an mir hängen und auf der anderen an ihrem Stock. Ihr Gesicht ist zu mir aufgehoben, sie befragt mich mit leuchtendem Blick, ich lächle sie an und sie glaubt mir. Ich bin ihr mütterlicher Vater.

Und wenn sie gleich groß gewesen wäre wie ich? Oder grösser? ${ }^{28}$

25 Vgl. Jacques Rancière. Politik der Bilder. Übers. von Maria Muhle. Zürich und Berlin: Diaphanes, 2009, S. 14-19.

26 Roland Barthes. Die helle Kammer. Bemerkungen zur Photographie. Übers. von Dietrich Leube. Frankfurt a. M.: Suhrkamp, 1985, S. 100.

27 Rudolf Eisler. [Art.] „Entelechie“ [1904]. Wörterbuch der philosophischen Begriffe. https:// www.textlog.de/3935.html (Zugriff am 09.11.2020).

28 Hélène Cixous. Meine Homère ist tot... Übers. von Claudia Simma. Wien: Passagen, 2019 [2014], S. 11. 
Die literarische Sterbebegleitung gerät zu einer regelrechten Zerstäubung von Identität, die keinen festen Grund und Boden mehr hat (,ich bin ihr mütterlicher Vater"). In seiner sinnhaften und sinnlichen Polyvalenz arbeitet der Text das Sterben der eigenen Mutter beständig durch, durchquert die Zeiten. Durch diese entschiedene Anachronie versucht er ihn als Prozess der Permanenz erlebbar werden zu lassen, der in der Gestalt eines dauernden Aufschubs erst als futurum exactum abgeschlossen sein wird („wo meine Mutter geweilt haben wird“). Die Stimmen von Mutter und Tochter verbinden, die Positionen vermischen sich bis hin zur Umkehr der Rollen von Mutter und Kind. Gegenwart, Vergangenheit und Zukunft sind ineinander verschlungen, als ob gerade diese Verquickung das endgültige Herausgleiten aus der Zeit, ja das Sterben verhindern könnte. Die dahinterliegende darstellungsästhetische Strategie ist eine écriture, die sich vehement gegen Linearität und Diachronizität, mithin gegen das Ende der Zeit stemmt, indem sie das Archiv der Vergangenheit mit der Vorkünftigkeit des Sterbens zu einem All-Raum verschaltet, der das Sterben sogar rückwirkend im Schreibprozess noch einmal aufschiebt. „Gegenwärtiges Leben und zukünftiger Tod verschmelzen“, wie Claudia Benthien mit Blick auf den Vanitas-Topos in der Barockdichtung analysiert, also auch hier, „in eine einzige, synthetisierte Zeit, wodurch ein unheimlicher, hybrider Seinszustand entsteht“" ${ }^{29}$

Cixous ruft ihre Mutter in der vollen Vieldeutigkeit von sprachlichen Konkordanzen als Ursprung des Erzählens (Homer) und darin als Mutter (mère) an (hörbar mit Interjektion als Ô Mère!), die durch die Homonymie mit dem Wort mer zusätzlich als fluides Wesen erscheint. Darüber hinaus scheint in dem Begriff homme (,homme-mère'), also ,Mensch“ und ,Mann“ durch, jedoch als grammatikalische Intervention (der eigentlich maskuline Name Homère wird hier zum Femininum: Homère est morte lautet der Titel im französischen Original), was das Maskuline hinter die weibliche, mütterliche Umformung zurücktreten lässt und die Mutter nicht nur zur Gedächtnisträgerin einer Epoche, sondern sogar zur Trägerin ihres eigenen Schreibens macht, dem sie nur nachfolgen, es aber nicht beginnen und letztlich auch nicht beenden kann. Damit spitzt sie den kulturgeschichtlichen Topos des Aufschubs zu und setzt die Weigerung, ein Leben sich vollenden zu lassen, als Erzählstrategie ein, die die Gedankenfigur der ,ewigen Wiederkunft des Gleichen“ in der Erfahrungsdimension der Dauer

29 Claudia Benthien. „,Vanitas mundi‘. Der barocke Vergänglichkeits-Topos in bildender Kunst, zeitbasierten Medien und Literatur der Gegenwart“. Frühe Neuzeit - Späte Neuzeit. Phänomene der Wiederkehr in Literatur und Künsten ab 1970. Hrsg. vom Nordverbund Germanistik. Bern u. a.: Lang, 2011. 87-108, S. 91. 
der logischen Sukzession von Anfang, Mitte und Ende gegenüberstellt. ${ }^{30}$ „Unendliche Zersprengung des Augenblicks, des Dings, des Jetzt“31 vollzieht sich mit Bernard Pautrat durch den Nietzscheanischen Gedanken der Wiederkehr. Die literarische Inszenierung funktioniert, prägnant formuliert, auf folgende Art und Weise: Gegen das Bildnis von der Mutter, das mit ihrem Tod konvergieren würde, gegen das punctum des Archivs, das das betrachtende Ich „vor einer Katastrophe, die bereits stattgefunden hat ${ }^{* 32}$ erschauern ließe, wird durch ein geschichtet-verschlungenes Zeitkonzept von vergangenen, möglichen und gegenwärtigen Jetztmomenten in der Tätigkeit des immerzu wiederhervorholenden Schreibens ,reine Dauer، in Anschlag gebracht.

Solches Schreiben ist ein forciertes Spiel mit dem Phänomen Zeit, das seinerseits nichts anderes als ein wahrnehmungsästhetisches ,Spiel um Zeit‘ ist. Die Geste des Aufschubs ist ihm ebenso deutlich eingeschrieben wie diejenige des Entzugs. Durch Techniken der Wiederholung, Praktiken des Aufsammelns und Einwebens von großen und kleinen Geschichten, ist sie vergebliche Arbeit am Archiv. Das archivarische „Noema des ,Es-ist-so-gewesen“"33, das in Wahrheit weit über die Sphäre der Fotografie hinausreicht und das Vergangene in eine diskrete Logik des Eigentums einspeist, insofern es auf dessen Unveränderlichkeit beharrt, wird in eine Spannung von Halten und Entfernen, von Fixieren und Loslassen überführt. Auf diese Weise erfährt der von Heidegger in die Diskussion eingeführte ,Sachverhalt' zwischen Sein und Zeit, Vergänglichkeit und Ewigkeit eine performative und medienreflexive Verhandlung in der Schrift. Das komplexe Verhältnis zwischen dem Vergehen, das dem Vanitas-Topos mitsamt dem ihm zugehörigen lebenspraktischen Imperativ des Todesbedenkens einerseits eingeschrieben ist und dem Prozess des umwandelnden ,Wieder-hervor-Holens` andererseits, transzendiert dabei letztlich in eine eigentümliche Form der Homöostase, eines Stillstellens von Bewegung in der unablässigen Wiederholung.

30 Mit dieser Idee verband Friedrich Nietzsche - im Unterschied zur Nietzsche-Rezeption im 20. Jahrhundert - noch eine Dystopie: „Das grösste Schwergewicht - Wie, wenn dir eines Tages oder Nachts, ein Dämon in deine einsamste Einsamkeit nachschliche und dir sagte: Dieses Leben, wie du es jetzt lebst und gelebt hast, wirst du noch einmal und unzählige Male leben müssen“. Friedrich Nietzsche. „Die fröhliche Wissenschaft“. Sämtliche Werke. Kritische Studienausgabe 3. Hrsg. von Giorgio Colli und Mazzino Montinari. München: dtv, 1988. 343-651, S. 370 .

31 Bernard Pautrat. „Nietzsche, medusiert“. Nietzsche aus Frankreich. Hrsg. von Werner Hamacher. Hamburg: Europäische Verlagsanstalt, 2007. 165-182, S. 171.

32 Barthes 1985 (Anm. 26), S. 106.

33 Ebd., S. 87. 


\section{Spiele um Zeit}

Die menschliche Dauerklage über „die auf Widerruf gestundete Zeit“34 gerät vor der Folie dieser exemplarischen Formen zeitgenössischer Vanitas zur performativen Trauer über den Verlust dessen, was man ohnehin nie besessen hat und doch von der Rhetorik des Eigentums beherrscht wird. Die barocke Geisteshaltung war in ihrer religiösen Codierung noch umfassend von der göttlichen Heteronomie durchzogen, die das menschliche Leben in den Händen hält. Ganz in diesem Sinne entdeckte auch Franz Kafka im Begriff ,sein“ ein bestürzendes Homonym: „Das Wort ,sein“ bedeutet im Deutschen beides: Da-Sein und IhmGehören.“" ${ }^{35}$ Zeitgenössische Vanitas-Topiken stehen demgegenüber vorwiegend im Zeichen des Lamentos über ein Zeitregime, das als unerbittlich empfunden wird. Die knappe Ressource, die in der ubiquitären Bemerkung, keine Zeit ,zu haben', so unmittelbar wie ungenau angesprochen wird, hat das Sein in der Zeit längst einer kapitalistischen Redefigur unterworfen. Einzig noch die angemessene Nutzung von Zeit, so scheint es, vermag gegenwärtig die Kränkung ihres Entzugs ,für kurze Zeit‘ zu kompensieren. Der Blick auf die künstlerische Renaissance des Nachdenkens über die Flüchtigkeit des Lebens muss die zeitgenössische Logik von Ökonomie in nahezu allen Lebensbereichen bis hin zur Freizeitgestaltung ebenso berücksichtigen wie den Wegfall des verbindlichen theologischen Rahmens historischer Vanitas-Diskurse mit seinen dezidiert mahnenden und konsolatorischen Funktionen.

Selbstredend zeigt demnach nicht jedes Symbol, vom Totenschädel bis zum Blumenstrauß, nicht jede Meditation über das Ende des Lebens per se die piktorale, narrative oder performative Wiederaufnahme barocker Codes an. Auch mag das sprichwörtliche Spiel der Zitate die postmoderne Praxis des autoreflexiven, nicht-referentiellen Zeichengebrauchs verkörpern, die Bedeutung und Bezug wuchern und absichtsvoll ins Leere laufen lässt. Aus den gewandelten moralischen und ästhetisch-medialen Bedingungen allerdings wie Christine Buci-Glucksmann pauschal den Schluss zu ziehen, dass die zeitgenössische Kunst nur mehr „vanités secondes ${ }^{\text {36 }}$ produziere, in denen der Tod lediglich in neutralisierter Form prä-

34 Ingeborg Bachmann. „Die gestundete Zeit“. Die gestundete Zeit. Gedichte. München: Piper, 2011, S. 22.

35 Franz Kafka. Nachgelassene Schriften und Fragmente II. Hrsg. von Jost Schillemeit. Frankfurt a. M.: Fischer, 1992, S. 56.

36 Christine Buci-Glucksmann. „Les Vanités secondes de l'art contemporain“. Les vanités dans l'art contemporain. Hrsg. von Anne-Marie Charbonneaux. Paris: Flammarion, 2010. 53-87, S. 54. 
sent sei, ${ }^{37}$ ist differenzierungsbedürftig. Ihr ,Wiederlesen' im Barthes'schen Sinne scheint hingegen geboten. Die Relektüre widersteht mit Barthes den „kommerziellen und ideologischen Gewohnheiten unserer Gesellschaft [...], die es gerade nahelegt, die Geschichte ,wegzuwerfen', sobald sie konsumiert (,verschlungen') worden ist“, und „bewahrt den Text vor der Wiederholung“, indem sie, selbst im Zeichen der Vergänglichkeit, niemals dieselbe Geschichte noch einmal erzählt. ${ }^{38}$

So zeigen gerade die vielgestaltigen Vanitas-Rekurse zeitgenössischer performativer und installativer Praktiken - im intermedialen Spannungsfeld von Fotografie, Film und Video über Literatur und Musik - eine auffallende Tendenz zur medialen Reflexion des paradoxen temporalen Verhältnisses von Endlichkeit und Ewigkeit, von nunc currens und nunc permanens. Besonders markant ist die Performanz einer Ästhetik des Entzugs, die das prozesshafte Vergehen gegen eine Vorstellung von bildhaft geschlossener Totalität ausspielt. Die tradierte, verlässlich codierte Symbolik von Vanitas-Objekten tritt darin in den Hintergrund. Stattdessen liefert die Aporetik des momenthaften ,Jetzt ${ }^{\star}$ und ,Nicht-mehr' im Echoraum der historischen Vanitas-Codes ein künstlerisches Agieren ,auf zweiter Stufe“39. Das Spiel ,auf' Zeit, das wir Leben nennen, wird zu einem gleichermaßen darstellungsund wahrnehmungsästhetischen Spiel ,um‘ Zeit.

Im Gegensatz sowohl zur skizzierten Schichtungstechnik, die unterschiedliche Jetztpunkte, disparate Zeitkonfigurationen in ,ein' fotografisches Tableau setzt und dadurch konfligierende Zeiten synthetisiert, als auch zur literarischen Konstruktion einer achronisch auskomponierten All-Zeit, steht gegenwärtig eine auffällige Vielzahl an künstlerischen Formen, die Zeit thematisieren, ohne sich auf die vordergründige Dialektik eines verlusthaften Vergehens und Neuentstehens zu berufen. Angesprochen sind damit temporale Gefüge, die das Vergehen von Zeit als spezifische Konstellation von Dauer thematisieren. Einschlägig dafür sind installative Ereigniskonzepte, die gleichsam als dauernde Abbilder eines „bewegte[n] Bild[es] der Ewigkeit“40 die Werkprogrammatik des geschlossenen Ganzen ostentativ unterlaufen. Indem sie sich dem dramaturgischen Nexus von Anfang, Mitte und Ende widersetzen, torpedieren sie die Vorstellung des geschlossenen, werkhaften Gan-

37 Vgl. ebd., S. 54-55: „[L]es Vanités secondes sont des allégories indifférentes, où la mort est présente et neutralisée, dans une distance réflexive propre au primat de la surface et de la superficie.“ 38 Roland Barthes. S/Z. Übers. von Jürgen Hoch. Frankfurt a. M.: Suhrkamp, 2007, S. 20.

39 Vgl. Gérard Genette. Palimpseste. Die Literatur auf zweiter Stufe. Übers. von Wolfram Bayer. Frankfurt a. M.: Suhrkamp, 1993.

40 Platon. „Timaios“. Übers. von Franz Susemihl. Sämtliche Werke in drei Bänden III. Hrsg. von Erich Loewenthal. Darmstadt: Wissenschaftliche Buchgesellschaft 2004. 91-191, S. 116, 37 d 5. 
zen zugunsten einer dezidierten Ereignisästhetik. Die dem Menschen vorenthaltene Erfahrung der Ewigkeit, von der die historischen Vanitas-Reflexionen im Gestus der Melancholie ex negativo durchzogen sind, wird in zeitgenössischen durativen Konzepten performativ überaffirmiert. Die ästhetische Erfahrung des Augenblicks und mit ihr des Entzugs wird so selbst thematisch.

\section{(Über-)dauernde Situationen}

Das darstellungs- und wahrnehmungsästhetische Ausloten von Dauer erfolgt seit dem ausgehenden 20. Jahrhundert vermehrt auch durch das explizite Ausstellen und Ausagieren des Dauerns in theatral-installativen Situationen. Die Überlänge, die musikalischen Kompositionen wie Morton Feldmans 2. Streichquartett, dem mit mehr als sechs Stunden Aufführungsdauer längsten in der Geschichte der Gattung, Karlheinz Stockhausens 29-stündigen Opernzyklus LICHT oder dem konkret am Konzept einer ,ewigen Aufführung' ausgerichteten $O R G A N^{2}$ / ASLP von John Cage zugrunde liegt, ${ }^{41}$ ist für eine beträchtliche Anzahl an theatral-performativen Arbeiten konstitutiv geworden, in denen die negative Zuschreibung der ,Überlänge‘ für kunstphilosophische Aussagen funktionalisiert wurde.

So brachte die jüngere theatrale Kunstgeschichte mit performativen Mitteln eine Vielzahl an durativen Formen hervor, die einen produktiven Widerspruch zur genuinen Kategorie des Ephemeren konstellieren. Von Dauer sind dabei nicht nur die ekstatischen Erfahrungsdimensionen der Akteur`innen, wie sie in zahlreichen Erscheinungsformen der Durational Art seit den 1970er Jahren erprobt wurden, in denen Künstler`innen vornehmlich ihr eigenes temporales Ausharren in Situationen der Askese und des Entzugs zur Allegorie ihres eigenen Überdauerns funktionalisierten, sondern auch diejenigen der Zuschauer^innen und Zuhörer^innen. Die Zeit der Rezipient`innen und mit ihr die Fähigkeit, lange, mitunter bis zum Überdruss weilen zu können, wird herausgefordert, an ihre Grenzen gebracht. ${ }^{42}$ Wie lange die Aufführung dann aber für jede*n Einzelne ${ }^{\star} n$ währt, wie lange also die Erfahrung ,den` Teilnehmenden tatsächlich ,dauert‘, ${ }^{43}$ wird

41 Vgl. Holger Schulze. „Sound Monuments. Eine Inversion der Vanitas in den Klangkünsten“. Paragrana. Internationale Zeitschrift für Historische Anthropologie 27.2 [Themenheft ,Vanitas. Reflexionen über Vergänglichkeit in Literatur, bildender Kunst und theoretischen Diskursen der Gegenwart‘. Hrsg. von Claudia Benthien und Victoria von Flemming] (2018): 231-246.

42 Vgl. Johanna Zorn. „Lange weilen können. Maßlose Dauer und begrenzte Zeiterfahrung in einer Ästhetik des Durativen“. Forum Modernes Theater 31.1-2 (2020): 26-38.

43 Für den Hinweis auf die altertümliche Formulierung, wonach ,jemanden etwas dauert", wenn man etwas erträgt oder unter etwas leidet, danke ich Claudia Benthien. Vgl. hierzu auch 
ihnen explizit selbst überlassen. Denn die Situationen, die durative Ereignisse eröffnen, gehen mit dem Programm einher, dass die wahrnehmenden Individuen an irgendeinem Punkt in ihren Verlauf eintreten und in einem selbstgewählten Moment wieder austreten können. Subjekte bestimmen ihre Ein- und Austrittszeitpunkte in den und aus dem Verlauf also selbst, während die Aufführung weiter andauert. Innerhalb der ästhetischen Idee von Installation, die damit angesprochen ist, zeichnen sich die Settings durch die formale Weigerung aus, einen Anfangs- und Endpunkt zu haben und thematisieren so konkret die Differenz zwischen dem zeitlichen Verlauf des Kunstwerks und der Zeit der ästhetischen Erfahrung. ${ }^{44}$ Das Strukturmerkmal der Unermesslichkeit entzieht sich für jedes einzelne Subjekt seinem vollständigen temporalen Erfassen, seinem Dabeisein, und sei es nur, weil über die Dauer von Stunden die Konzentration nachlässt, sich Müdigkeit einstellt oder der Gang zur Toilette nötig wird.

Im Unterschied etwa zu Wiederholungsschleifen der Videokunst im Umfeld der Minimal Art wie Bruce Naumans Bouncing in the Corner, No. 1 (1968) oder der filmischen Explikation des Verstreichens von Echtzeit in Andy Warhols Empire (1964), deren spezifische Charakteristika der Wiederholbarkeit und Zirkularität tatsächlich endlose Vorführungen ermöglichen, erscheint die Dialektik von Unendlichkeit und Begrenzung in der theatralen Aufführungspraxis durch die performativen Dispositive physischer Ko-Präsenz und Flüchtigkeit zugespitzt. Der „epistemologische[] Effekt [...] als Zeitmaschine“ ${ }^{\text {45 }}$ taucht in den ästhetisierten Dauerkonfigurationen nichtsdestoweniger als künstlerisches Veto gegen die Bestrebung auf, den Dingen ein Ende zu setzen und vermag die existentielle Erfahrung einer vorweggenommenen Trauer über das Ende im künstlerischen Als-ob zu thematisieren. Zum strukturellen Prinzip erhoben wird dadurch ihrerseits die Wahrnehmungsdimension einer Unermesslichkeit, die allerdings notwendigerweise begrenzt bleiben muss.

Ohne Unterlass zu spielen, ist das leitende künstlerische Prinzip des britischen Performance-Kollektivs Forced Entertainment. Dieses hat sich seit den 1990er Jahren auf Aufführungen spezialisiert, die auf der Basis vereinbarter Spielregeln repetitive Strukturen errichten und improvisierte Verläufe in Szene setzen, die

die Bemerkung, dass „Dauern eine vergrößernde Wiederholungsform [ist], und bedeutet: Jemanden sehr weich machen, innig rühren. Das dauert mich sehr, will also eigentlich sagen: es macht mein Gemüth sehr weich." Johann August Eberhard. Versuch einer teutschen Synonymik in einem kritisch-philosophischen Wörterbuche der sinnverwandten Wörter der hochteutschen Mundart 1. Halle: Ruffsche Verlagsbuchhandlung, 1826, S. 364.

44 Vgl. Juliane Rebentisch. Ästhetik der Installation. Frankfurt a. M.: Suhrkamp, 2003, S. 196. 45 Christa Blümlinger. „Vom Nutzen und Nachteil der Schleife für die Montage“. montage AV 20.2 (2011): 149-160, S. 150. 
nicht nur oft den Anschein haben, endlos dauern zu wollen, sondern mit bis zu 24 Stunden Spieldauer, wie etwa im Fall von Who Can Sing A Song to Unfrighten $M e$ ? (1999), tatsächlich häufig überbordende Längen haben. Auf der Basis szenischer Strukturen, die das Werkkonzept der Geschlossenheit negieren und stattdessen vornehmlich dem Prinzip der variierenden Wiederholung verpflichtet sind, konterkarieren die Arbeiten nicht nur konventionelle spannungsdramaturgische Prinzipien von Zuspitzung und Retardierung, sondern spielen qua ihrer konsequenten Ästhetik der Unabgeschlossenheit relativ offensichtlich mit dem pars pro toto für die Ewigkeit. Die Dialektik von Vergänglichkeit und Ewigkeit ist ihnen eingeschrieben, ohne den Tod als Sujet zu thematisieren. Im Gegenteil, die Performances sind dezidiert unterhaltsam, komisch, in ihrer ironischen Subversion theatraler Vorschriften bisweilen hintersinnig.

Eine dieser Arbeiten ist der Geschichtenmarathon And on the Thousandth Night, der seit dem Jahr 2000 seinerseits gefühlt endlose Aufführungen erfuhr. Dort wird das künstlerische Prinzip der installativen Endlosschleife, des Loops, wie es aus der Video- und Medienkunst bekannt ist, auf die Theaterbühne transferiert und als Erzählen, das niemals zu einem Ende gelangen kann, szenisch transponiert: Männer und Frauen, gekleidet wie Könige und Königinnen mit roten Samtumhängen und Pappkronen, machen sich hier daran, in die Archive des kollektiven Gedächtnisses hinabzusteigen, um dort bekannte Geschichten hervorzuholen und neue zu erfinden. Dabei gibt es eine einzige szenische Verabredung: Eine ${ }^{\star} \mathrm{r}$ der Performer ${ }^{\star}$ innen beginnt mit den Worten „Once upon a time there was a king“ und erzählt so lange weiter, bis ein ${ }^{\star} e$ andere ${ }^{\star}$ r Performerin die Geschichte unterbricht, um wiederum mit der gleichen Einleitungsfloskel anzuheben, die Geschichte weiterzuerzählen, in eine andere Richtung zu treiben, sie zu ironisieren, oder auch nur den Wettstreit um die bessere Anekdote, die eindrücklichere Performance aufzunehmen. Dieses Prinzip wiederholt sich über die gesamte Spieldauer von sechs Stunden. Gelegentlich machen die Performer*innen Pausen, trinken Kaffee oder schlafen, denn auch für sie vergeht Zeit, stellt sich Müdigkeit ein. Als Zuschauer^in wiederum ist man gespannt, wie lange es dauert, bis die gerade gehörte Geschichte im Zeichen des kollektiven Sprechens durch eine ${ }^{\star} n$ andere ${ }^{\star} n$ Sprecher ${ }^{\star}$ in in eine neue Richtung gelenkt wird. Allzu oft bricht sie an der spannendsten oder wirkungsvollsten Stelle ab.

Das strenge Formkonzept mit seiner immanenten Ästhetik der Unterbrechung etabliert über die Dauer der Aufführung die große Erzählung von Zeit, genauer des Vergehens von, in und mit Zeit, das niemals abbricht, innerhalb dessen man aber unversehens abberufen werden kann. Es korrigiert auf diese Weise die Vorstellung vom Ende des Lebens in Richtung eines unvermittelten Abgebrochenwerdens. Aus den Fragmenten, aus dem Akt des gegenseitigen Zuhörens und Weiterspinnens entsteht eine performative Situation der Dauer. Ent- 
scheidend dabei ist, dass die Aufführung, die faktisch zwar zu einem bestimmten Zeitpunkt beginnt und endet, ein Aufführungsdispositiv etabliert, das weder Anfang noch Ende hat. Den Rezipient*innen wird explizit die Möglichkeit gegeben, ad libitum zu kommen und zu gehen. Mit dieser Freiheit zur temporalen Beständigkeit und Unterbrechung der ästhetischen Erfahrung errichtet die Erzählperformance ein nahezu minimalistisches Konzept von Dauer, das wesentlich auf der Ambivalenz der temporalen Struktur der Wiederholung beruht. Das Prinzip der Iteration, das mit seiner offenkundigen Geste des ,Noch-einmal-von-vorne-Beginnens ‘ immer schon auf das nicht Einholbare im Archiv verweist, gerät zur Grundlage dieser performativen Verhandlung von Dauer. Entsprechend wird auch das Vergehen von Zeit selbst thematisch und steht auf widersprüchliche Weise im Zeichen „eines Flehens um Ewigkeit“46. Durch die kreisförmige temporale Bewegung, die ein Zu-Ende-Erzählen aller partikularer Geschichten entschieden verhindert, wird der „Vergänglichkeitswert“, den Sigmund Freud als „Seltenheitswert in der Zeit“ und darin als wertsteigernd bestimmte, performativ aufgehoben, um ihn nach dem Prinzip der Differenz in der Wiederholung gleichzeitig umso entschiedener ins Spiel zu bringen. ${ }^{47}$

Damit rückt zugleich die Deckung von künstlerisch gestaltetem und tatsächlichem Vergehen von Zeit in den Mittelpunkt. Eine solche durative theatrale Ästhetik führt uns, da sie irgendwann anfängt und irgendwann wieder aufhört, in explizit anti-aristotelischer Manier vor Augen, dass die Geschichten unseres Lebens, indem sie Anfang, Mitte und Ende haben, die wir selbst bestimmen können, Phantasmen sind. And on the Thousandth Night zeigt in seiner dramaturgischen Verdopplung einer lebensweltlich virulenten Zeitstruktur, also dem tatsächlichen fortwährenden Vergehen von Lebenszeit, die immer so weiter gehen könnte und irgendwann doch endet, einen ästhetisierten Versuch über die Unermesslichkeit, die Ewigkeit selbst. Schon Scheherazade, die offensichtlich den entscheidenden Intertext liefert, wusste in ihrem Erzählen von tausend und einer Geschichte von der Möglichkeit schier endlosen Weitererzählens, um auf diese Weise dem drohenden Tod zu entgehen.

\section{Das Standbild der Kunst}

Unermesslich bis zur Zumutung ist auch Romeo Castelluccis im Jahr 2015 erstaufgeführtes Projekt Le Metope del Partenone. Mit einer Spieldauer von einer knappen

46 Manfred Schneider. Die erkaltete Herzensschrift. Der autobiographische Text im 20. Jahrhundert. München: Hanser, 1986, S. 14.

47 Sigmund Freud. „Vergänglichkeit“. Gesammelte Werke X. Hrsg. von Anna Freud. London u. a.: Imago, 1946. 358-361, S. 359. 
Stunde taugt die Arbeit auf den ersten Blick schwerlich als signifikantes Beispiel einer durativen Ästhetik. Allerdings existiert auch hier ein einfaches wie strenges Formgesetz von Wiederholung, das potentiell unendlich weitergehen könnte. Nur ist es in diesem Fall das Sterbenmüssen in all seiner Kontingenz, wie es sich tagtäglich an vielerlei Orten ereignet, dessen unentrinnbar beständige Wiederholung im Modus der performativen Affirmation das iterative Strukturgesetz der Aufführung liefert: Ein Mensch wird Opfer eines Unfallgeschehens, er erlebt seinen Todeskampf und jede Hilfe kommt zu spät. Castelluccis theatrale Reflexion über diesen alltäglichen Vorgang stellt den Prozess des Sterbens nach und wiederholt ihn permanent, sodass ein minimalistisches szenisches Konzept entsteht: Sechsmal betreten eine Darstellerin oder ein Darsteller eine riesige Halle, in der sich das Publikum frei bewegt, Maskenbildnerinnen in weißen Kitteln präparieren ,die von der Zeit gesegnete' Person mit Theaterblut, bevor sie zu Boden geht. Der oder die Betroffene fängt an zu zucken, winselt, schreit oder krümmt sich vor Schmerzen. Dem einen quellen die Gedärme aus dem offenen Bauch, der nächsten verbrennt Säure den Körper, wieder eine andere erwacht neben ihrem abgerissenen Unterschenkel schreiend aus der Ohnmacht. Was dann passiert, wiederholt sich ebenso in jeder einzelnen dieser aussichtlosen Unfallminiaturen: Stets kündigt das Signalhorn das Erscheinen eines tatsächlichen Rettungsautos an. Stets bleiben die Reanimationsversuche der wirklichen Sanitäter*innen und Notärzt*innen an den dargestellten Opfern erfolglos. Stets stellt das Herzmessgerät seine Tätigkeit ein. Stets ertönt der unheilvolle Ton, der das Ausbleiben der Herzfrequenz akustisch besiegelt. „Für jede und jeden der zum Tode hin Versehrten ist das Ende unumgänglich gleich: Ein weißes Leintuch wird über den oder die Verewigte(n) gelegt.“48 Das Rettungsauto fährt ab.

Im Verlauf der Wiederholung dieses Prozesses ahnt man als Rezipient`in schnell, dass der Abend diesem strengen Formgesetz treu bleiben wird. Allerdings stehen die im Spiel Verstorbenen allesamt nach ihrem Tod wieder auf und verlassen schreitend die Halle, so als wollten sie noch einmal betonen, was ohnehin offensichtlich ist: Dies ist alles nur ein Spiel, gestorben wird hier nur im Akt der Kunst. Bevor sie die Halle verlassen, verharren sie, den Blick auf das Publikum gerichtet, wie Statuen an einer Wand, auf der rätselhafte Aussagesätze geschrieben stehen: „Ich bin nie gewesen, aber im Werden begriffen“ heißt es da etwa, „Ich habe keinen Körper, aber du kannst mich sehen“ oder „Ich bin immer der Anfang des Endes“. Alle geben Hinweise auf die immer gleiche Frage: „Wer bin ich?“

48 Ronald Pohl. „Blutige Festwochen-Performance: Tot zu sein bedarf es wenig“. Der Standard (08.06.2019). https://www.derstandard.at/story/2000104577778/blutige-festwochen-per formance-tot-zu-sein-bedarf-es-wenig (Zugriff am 09.11.2020). 
Dem Menschsein, so wird deutlich, droht der plötzliche Tod immer. Vor dem erhabenen Sterben, dem ,Jetzt, das geschieht ${ }^{69}$, bleibt wohl nur die Einsicht in die Faktizität einer „Katastrophe, die unablässig Trümmer auf Trümmer häuft “50. Auf diese blickt auch der ${ }^{\star}$ die Teilnehmer*in dieser unerbittlich nüchternen Meditation über die unhintergehbare Tatsache des Sterbens. Einzig die Standbilder der Kunst und mit ihnen der Rätselcharakter des Lebens, vermögen da noch Hoffnung zu geben. Und so fokussiert Le Metope del Partenone jenen Moment der Kunst, in dem Vergänglichkeit sich in Sinn verwandelt. Das titelgebende, antike Figurenfries des Parthenon-Tempels in Athen, das von mythischen Kämpfen gegen Giganten, Amazonen und Kentauren erzählt, liefert die Quelle für die ästhetische Reflexion über Permanenz und Vergänglichkeit. Der vergeblich um sein Überleben kämpfende Mensch muss erst zu Kunst werden, um Ewigkeit zu erlangen.

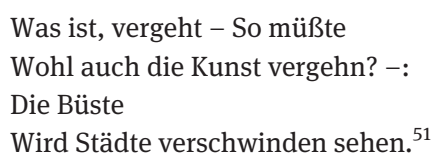

So heißt es in Théophile Gautiers Gedicht „Die Kunst“ (1857). Die Allusion an die Denkfigur der ,langen Kunst‘, die der lateinische Aphorismus vita brevis, ars longa prägnant formuliert, ${ }^{52}$ wird bereits in der Frühen Neuzeit gegen die Kürze des Lebens aufgeboten und übernimmt die tröstende Funktion einer Verewigung all dessen, was sterben muss. Das Zeitkonzept der fortwährenden Wiederholung, das Castellucci mit seinem Ensemble für die Unentrinnbarkeit des Vergehens aufbietet, spitzt diese Vorstellung von der ewigen Kunst zu einer streng repetitiven Ästhetik des Durativen zu: Zeitlichkeit als Begrenzung des Lebens evolviert gerade aus der

49 Vgl. Barnett Newman. „The Sublime is Now“. Selected Writings and Interviews. Hrsg. von John P. O’Neill. New York, NY: Knopf, 1990. 170-173; sowie die zentrale Rezeption von Newmans Vorstellung vom Erhabenen als instant présent durch Lyotard: Jean-Francois Lyotard. „Das Erhabene und die Avantgarde“. Übers. von Clemens-Carl Härle und Heike Rutke. Merkur 38.2 (1984): 151-164.

50 Walter Benjamin. „Über den Begriff der Geschichte“. Gesammelte Schriften I,2. Hrsg. von Rolf Tiedemann und Helmut Schweppenhäuser. Frankfurt a. M.: Suhrkamp, 1980. 691-704, S. 697.

51 Théophile Gautier. „Die Kunst“. Emailen und Kameen. Übers. von Otto Hauser. Weimar: Duncker, 1919. 35-36, S. 36.

52 In der bekannten Fassung Senecas, wonach „vitam brevem esse, longam artem“, wird der Satz Hippokrates (,ille maximi medicori“) zugeschrieben. Seneca. „De brevitate vitae/Die Kürze des Lebens“. Schriften zur Ethik. Die kleinen Dialoge. Übers. und hrsg. von Gerhard Fink. Düsseldorf: Artemis \& Winkler, 2008. 552-611, S. 552. 
Wiederholung, die ihrerseits aber unendlich so weitergehen könnte. Und so zeigt diese Arbeit, wie dauernde Formen der zeitgenössischen performativen Kunst im Allgemeinen, in signifikanter Weise ihren kunstphilosophischen Charakter. Die der Idee nach einer nicht enden wollenden ästhetischen Erfahrung des Durativen, die das Immergleiche potenziell ad infinitum durchspielt, und selbst in der Dauer einer Stunde theoretisch wie performativ wirksam werden kann, negiert ostentativ den Prozess von Zeitlichkeit als Vergehen, den sie dezidiert thematisiert, und kreiert ein prozessual bildnishaftes Konzept von Ewigkeit. Die Zeitenthobenheit der durativen Kunst liegt letztlich allerdings nicht in ihrer festen Gestalt, also dem gleichsam versteinerten Material, wie es Gautier am Ende seines Textes nahelegt und wie es Castellucci als szenische Transformation des Ephemeren in die Stein gewordene Bedeutung zitiert. Vielmehr artikuliert sie sich in ihrem selbst auferlegten Gesetz der endlosen Wiederholung, die das ewige Prozessgeschehen der Unendlichkeit reflektiert, indem sie es als Struktur in das ästhetische Geschehen selbst hereinholt. Konzepte von Ewigkeit werden auf diese Weise in durativen Arbeiten errichtet, die nur in Ausschnitten zugänglich sind und gerade deshalb mit der totalen Gedankenfigur des großen Ganzen arbeiten, an dem wir als Rezipient`innen bekanntlich immer nur unvollständig, nur partiell teilhaben können.

\section{Abbildungsnachweis}

Abb. 1 helmutwimmer.net (C) Helmut Wimmer 